\title{
A global approach for plantar fasciitis with extracorporeal shockwaves treatment
}

\author{
Federico Giordani (1), Andrea Bernini (1), Hannes Müller-Ehrenberg (2), Carla Stecco \\ (3), Stefano Masiero (1)
}

(1) Department of Physical and Rehabilitation Medicine, University of Padua, Padua, Italy; (2) Private Clinic Orthopädische Privatpraxis Dr. Hannes Müller-Ehrenberg, Münster, Germany; (3) Department of Neurosciences, Institute of Human Anatomy, University of Padua, Padua, Italy

This article is distributed under the terms of the Creative Commons Attribution Noncommercial License (CC BY-NC 4.0) which permits any noncommercial use, distribution, and reproduction in any medium, provided the original author(s) and source are credited.

\begin{abstract}
Extracorporeal Shockwaves Treatment is considered an effective therapeutic option for plantar fasciitis, but the standard application in the medial insertion of the plantar fascia on the calcaneus has provided ambiguous evidences. In this case, a 63-year man with plantar fasciitis was treated in a 3-session program and Foot and Ankle Outcome Scale and Foot Functional Index questionnaires were chosen for the clinical outcome evaluation. The therapy was focused on the active trigger or myofascial points of the leg, thigh and pelvis in order to return the correct equilibrium of the myofascial system of the whole limb. The patient has already reported an improvement after the second session (FAOS: 76 vs 33, FFI: 85\%) which was confirmed in the third one and in the 1-month follow up (FAOS: 79, FFI: 6\%) Results suggest that plantar fasciitis may be due to proximal rigidity or tension of the fascia and a global approach using ESWT may have a similar or better outcome respect to the standard application.
\end{abstract}

Key Words: Extracorporeal shockwaves treatment (ESWT), plantar fasciitis, fascia, myofascial impairment.

Eur J Transl Myol 29 (3): 171-177, 2019

Plantar fasciitis is a common, chronic, musculoskeletal disorder characterized by pain in the region of the plantar fascia; the most affected area of knife-like pain is the enthesis of the fascia, in the medial-plantar region of the heel near the medial calcaneal tuberculum. ${ }^{1,2}$ Its onset is insidious, and, usually, not well associated to a specific incident or trauma. The symptoms usually start as an intermittent pain with the first steps of the day or after a long walk and, day by day, progresses to become persistent. ${ }^{3}$ The pain perceived by the patient is typically worse after periods of long sitting and it exacerbates during weight-bearing activities. ${ }^{4}$ Because of this typical presentation of the symptoms, the development of plantar fasciitis is thought to have a mechanical origin. ${ }^{1}$ The most supported theories suggest how flat feet and lower-limb biomechanics disorders can lead to a lowered medial longitudinal arch; in biomechanical terms, this situation is thought to create excessive tensile strain within the fascia, producing microscopic tears and chronic inflammation. ${ }^{5}$ While common clinical approach tries to justify the onset of plantar fasciitis through biomechanical factors that promote excessive tensile strain within the plantar fascia as paramount to the development of plantar fasciitis, ${ }^{6}$ scientific support for this premise is very limited. ${ }^{1}$ So, despite its common diagnosis, little is known about the pathogenesis of this painful syndrome. About $90 \%$ of patients are, as first approach, treated with nonsurgical measures. ${ }^{7-9}$ Usually non-operative treatments are bed rest, reduce weight, ${ }^{6}$ or nonsteroidal anti-inflammatory drugs, orthotics, physical therapy, exercise, stretching or local cortisone injection are the initial choices. ${ }^{2,10,11}$ Several scientific works focused on the application of Extracorporeal Shock Wave Therapy (ESWT). ESWT is a pulsed sound wave, characterized by short duration, high-pressure amplitude and relatively low tensile wave component, but the mechanism of ESWT on human tissues is not completely clear. However, during the last years, many evidences about their effects on animal and human tissues have been produced. It have been described both radial and focused ESWT in terms of regenerative medicine:12-14 Wang et al. demonstrated that ESWT induces neovascularization in the enthesis of the Achilles tendon, as confirmed by early release of angiogenesis-related 
markers, including Vascular Endothelial Growth Factor (VEGF), endothelial nitric oxide synthase (eNOS) and proliferating cell nuclear antigen (PCNA) in the tenocyte's tissue. ${ }^{15}$ Bosch et al. demonstrated that ESWT have a profound effects on collagen remodeling and turnover showing the induced disorganization in collagen matrix (on healthy equine tendon) and the upregulation of COL1 expression 6 weeks after ESWT, that may be indicative of a stimulus of repairing; ${ }^{16}$ similar results have been published by Sokubo et al.'s studies about the effects of shock waves (SW) on macrophages: low energy shock waves reduce the expression of the proinflammatory profile M1 macrophages and induce the expression of an anti-inflammatory profile M2, suggesting how ESWT could contribute to switch off inflammation and to promote regeneration of the tissue. ${ }^{17}$ The effects of ESWT on human tissues have been compared to other conservative or, better, mini-invasive therapies, as local Corticosteroid's injections, to treat plantar fasciitis: significant improvement in pain was observed both with ESWT and with corticosteroid injections in the short-medium term period. ${ }^{18}$ Furthermore, Shuxiang $\mathrm{Li}$ and his team concluded that patients treated with high intensity ESWT, compared to the group of patients treated with corticosteroid injections, experienced less pain during the treatment and had more rapid return to usual activities after treatment. ${ }^{19}$ In addition, the effectiveness of ESWT, compared to Non-steroidal anti-inflammatory drugs (NSAIDS), orthotics, physical therapy, exercise program and local cortisone injection, has been demonstrated also in the long term period. ${ }^{20}$ About their safety, very limited and transitory ESWT's side effects are described, if used to treat muscoloskeletal disorders: regional pain, reddening of the skin, petechiae, hematomas and hypestesia. ${ }^{19,21}$ For the treatment of plantar fasciitis, ESWT are usually the first choice in the common clinical practice. Several studies and reviews reported variable success rate in terms of pain reduction, from $55 \%$ to $88 \%$, compared to placebo, in short- or medium-period. ${ }^{4,22-26}$ Among all of these confirmations about the beneficial effects of ESWT on plantar fasciitis, many other studies have not found any difference between ESWT-treated group and placebo-treated group. ${ }^{6,27,28}$ Therefore, some authors found a recurrence rate of plantar fasciitis from $5 \%$ to $11 \%$ in the middle-long term follow-up..$^{20,29,30}$ In all of these studies ESWT have been directed to the area of maximum pain, localized through profound palpation of the plantar fascia in many areas. Since the fasciae of the lower limb are a continuum, ${ }^{31}$ proximal tensions could generate stiffness and pain to distal regions as plantar fascia. Based on this evidence, our aim is to analyze and treat plantar fasciitis with a global approach in order to return a correct tension in the entire fascial system of the lower limb. Accordingly, we shift the focus of the area to be treated with ESWT from the sole of the foot, strongly inflamed and painful area, to other proximal points of leg, thigh and pelvis. This report is an introductory case report of the first patient of our study.

\section{Case Presentation}

A 63-year-old man presented heel pain in the plantar face of the left foot. The obese but otherwise healthy man states that he has had the pain daily for months. He worked for more than 30 years as welder wearing safety boots for 8 hours a day. During the first medical examination, he referred to the clinician an unrelenting heel pain radiated along the Achilles tendon and posterior leg muscles, severe in the morning and increasing with load. In addition, he reported occasional swelling of the ankle but denied numbness or tingling. The patient indicated great difficulties to perform daily life activities (as walking, climbing stairs and getting up from chairs) due to the pain and felt worried about the stability of his ankle. On physical examination, his feet and ankle have no abnormalities, edema or skin changes. Sensibility was found to be conserved for the entire lower limb and there was no strength deficit. The patient presented strong pain during heel palpation, specially corresponding to the medial insertion of plantar fascia on the calcaneus bone. When he was asked to walk on his heel, the pain was too strong to be tolerated. Moreover, he reported a feeling of stiffness and articular limitation of the hip and knee during lower limb active mobilization. Both legs had no pulse or reflex impairment. Finally, the patient received a diagnosis of plantar fasciitis. The patient was addressed to a conservative treatment by using ESWT and a 3session program was assessed. The clinical condition was investigated by using Foot and Ankle Outcome Scale (FAOS) and Foot Functional Index (FFI). FAOS is a selfadministered survey instrument to assess the disability of the foot and ankle and associated pathologies based on 5 subsections (symptoms and stiffness, pain, daily living, sports and quality of life). FFI consists of 23 self-reported items divided into 3 subcategories based on patient values: pain, disability and activity limitation. The patient has to score each question on a scale from zero (no pain or difficulty) to 10 (worst pain imaginable or so difficult it requires help), that best describes their foot over the past week. The 2 scales were filled out before each session and 1 month follow up was scheduled: T0 was defined as the first session, T1 as the second session, $\mathrm{T} 2$ as the third session and T3 as the interview in the 1month follow up. For each treatment, 3 or 4 point of stiffness and tenderness in the impaired lower limb and in the pelvis were chosen and 1500 shocks (Piezowave 2; $5 \mathrm{~Hz}, 0.167 \mathrm{~mJ} / \mathrm{mm} 2$ ) for each one were given. This protocol has been chosen upon the observation that this amount of energy is needed to lose the densification in the fascial point. Moreover, very rare adverse reactions have been reported in the literature and the safety of the treatment has been widely demonstrated. ${ }^{19-21}$ The selection of points was based on Fascial Manipulation's principles by Stecco that describes 3 different planes of treatment of the fascial system of the limbs and pelvis; 


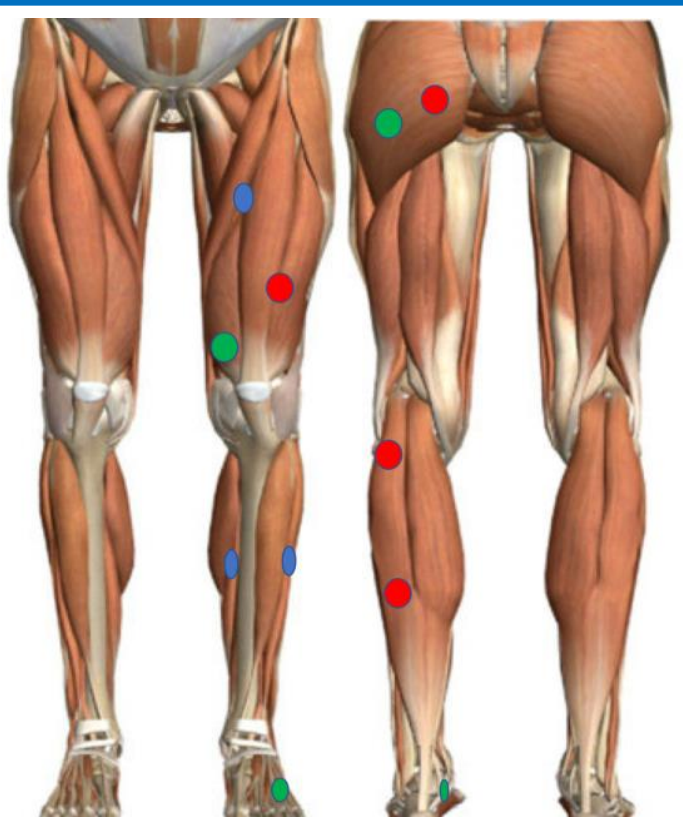

Fig 1. Visual representation of the points selected for the first session (red), second session (green) and third session (blue).

each one is composed by 2 antagonist sequences of fascial points (center of coordination, CC) that may be found impaired or densified and can create tension. For each treatment, one among the three planes has been chosen. This decision was based on: the sensations of stiffness and articular limitation reported by the patient himself; the physical examination of the active and passive articular movements; the research in every segment of the pelvis and lower limbs, through the palpation with the fingertip, the knuckle or the elbow, of the most densified (and, usually, the most painful) points of the fascia's sequences. During the three sessions, the following points of the left lower limb were selected: in T0 extra-pelvis, intra-genu, extra-pes, intra-pes; in T1

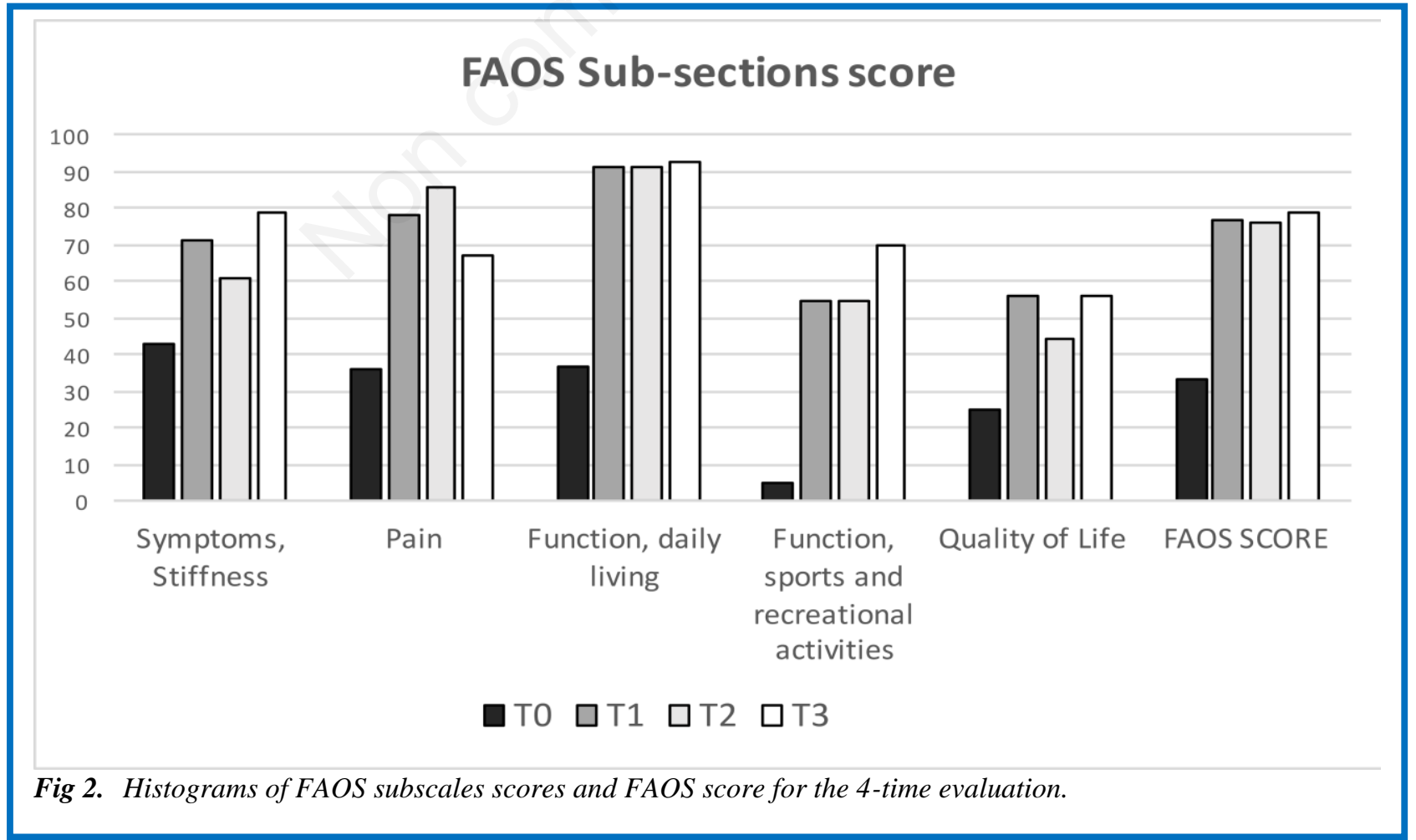




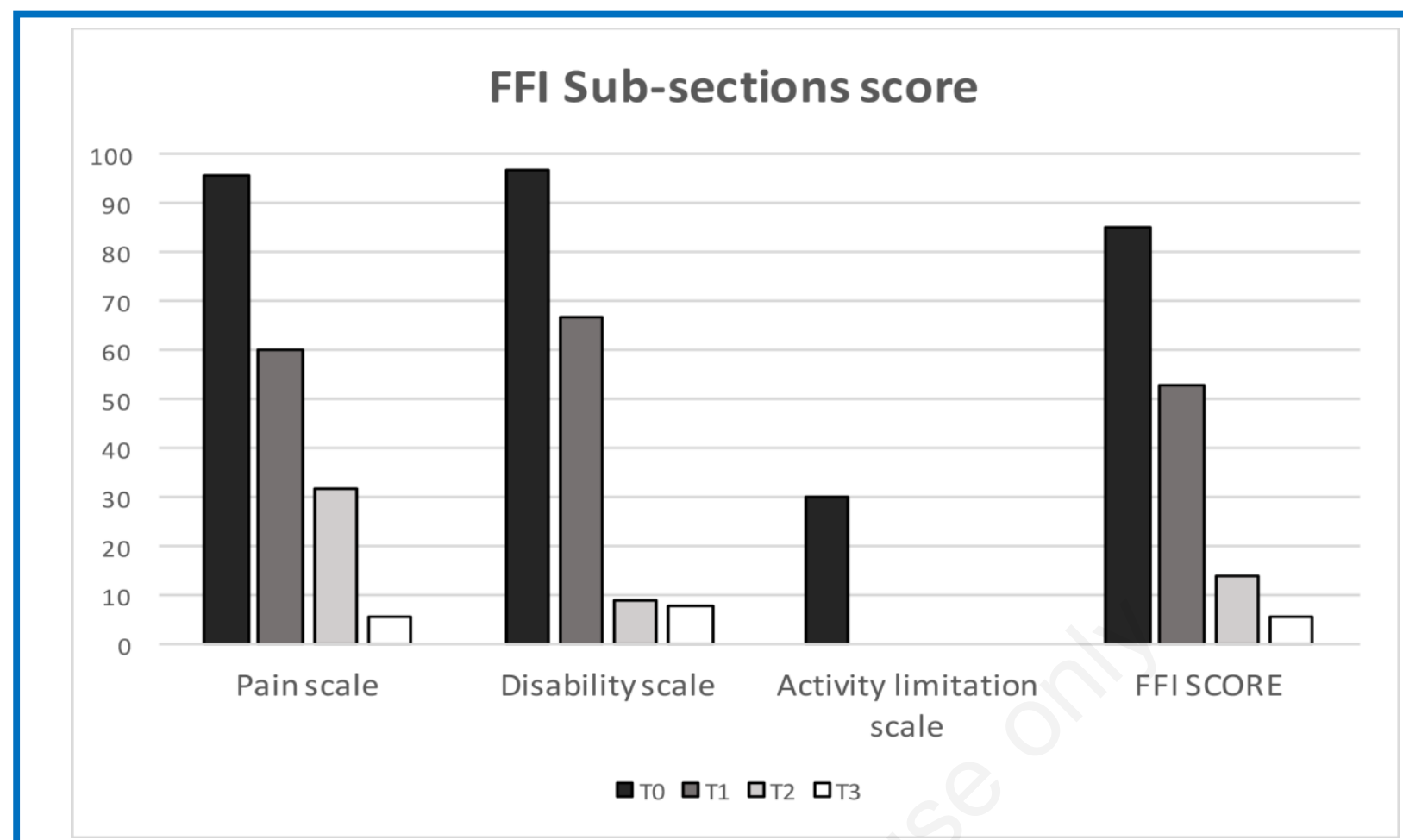

Fig 3: histograms of FFI subscales scores and FFI score for the 4-time evaluation.

extra-pelvis, extra-talus, intra-talus; in T2 retro-coxa, retro-latero-genu, retro-talus (according with Stecco's nomenclature). Figure 1 shows the points selected for this first report. In T0, FAOS score of 33 and FFI score of $85 \%$ were observed. Immediately after the application of $\mathrm{SW}$, the patient referred a significant reduction of the hip and knee sensation of stiffness and articular limitation. In $\mathrm{T} 1$, an improvement in FAOS (77 vs 33) and FFI (53\% vs $85 \%$ ) score was found and the previous sensations of stiffness and limitation in the knee and hip were not reported anymore. As regard the last session (T2), a FAOS score of 76 and FFI score of $14 \%$ were registered and knee and hip mobilization was observed to be similar to the previous sessions. In T3, improvement of symptoms was found to be confirmed (FAOS score $=79$; $\mathrm{FFI}=6 \%$ ). Subscales results are listed in Figure 2 and 3.

\section{Discussion}

In the last years, the fasciae and their properties are becoming of central importance to clinicians practicing in various conventional and alternative therapies. ${ }^{1}$ For the very first time, we applied ESTW in the altered myofascial points of the lower limb instead of in the site of pain according to fascial manipulation method. The rationale was to consider the myofascial continuity through the whole body. In fact, tension, tightness and strength of the plantar fascia doesn't depend only on foot's characteristics: plantar fascia is part of a more complex unit named, decades ago, "Achilles-calcaneusplantar System": ${ }^{32}$ there is a functional connection between the Achilles tendon and the plantar fascia through the posterior trabecular system of calcaneus, that would work as a big sesamoid, transmitting the force from the tendon to the fascia. The deep link between Achilles tendon and plantar fascia has been demonstrated, in terms of embryological development, also by a study conducted by Shaw et al. ${ }^{33}$ : they observed in the fetus a marked anatomic continuity between Achilles tendon and plantar aponeurosis, through a thickening of the perichondrium in the posterior part of the main body of the calcaneus. This continuity has been demonstrated also in adult people by Stecco et al. in 2013:34 plantar is more closely connected to the paratenon of Achilles tendon rather than to the Achilles tendon, through the periosteum of the heel. Therefore, the fascia cruris that wraps gastrocnemius muscle and the paratenon around the Achilles' tendon are continuous structures. ${ }^{35}$ As regard to muscular component, people with hamstring tightness are more subject to have plantar fasciitis in the corresponding foot. ${ }^{36}$ Furthermore, there are many proofs that muscular forces are transmitted through the fascia thanks to their fascial insertions. ${ }^{37-39}$ So, from a myofascial point of view, tension of the hamstrings, strongly connected to the fascia of the thigh, could be transmitted to the fascia cruris increasing the tension of the plantar fascia. Thus, it is likely that problems regarding proximal structures could generate impairment in the distal areas of the lower limb leading to inflammation and degeneration of plantar fascia 
sustained by usage and overload. It may also explain why some patients refer a proximal radiation of the pain that has the features of a myofascial pain more than a nervous one. ${ }^{40}$ In the literature, the standard protocol for ESWT includes the application in the pain site, mostly in the medial insertion of the plantar fascia, ${ }^{6,20,22,27}$ but there are evidences that associating ESWT to stretching exercises of the Achilles-calcaneus-plantar System plantar could be more effective than the treatment with ESWT alone; ${ }^{41}$ furthermore, Labovitz et al. ${ }^{36}$ suggested that stretching exercises should be extended also to the whole posterior muscular leg chain, to reduce tension and stiffness of the plantar fascia.

\section{Conclusions}

The application of ESWT in the insertional site of the plantar fascia alone can heal the local impairment but may not be sufficient to solve the primary cause of plantar fasciitis and may expose the patient to risk of recurrence. In this case, the application of shockwaves in the impaired myofascial points of the leg and pelvis aimed at restoring the sliding properties and the equilibrium in the myofascial system of the entire lower limb in order to return the correct function of the plantar fascia without treating the plantar fascia itself which is often found to be inflamed and painful. The major strengths of this paper are that we considered plantar fasciitis as a global alteration of the fascial system rather than a local impairment; it may solve recurrence and failure rate. Further studies are necessary to confirm those primary findings and a greater number of patients have to be involved: this is the major weakness of this research. If confirmed, these results could suggest a different explanation for etiopathogenesis of plantar fasciitis and may shift the focus from the plantar fascia itself to the more complex fascial system; it could help the physician to consider new solutions in order to solve the problem. Anyhow, this is the first published report of extracorporeal shock waves treatment for plantar fasciitis that takes into account a global approach to the pathology.

\section{List of acronyms}

$\mathrm{CC}$ - center of coordination

COL1 - anti-collagen 1 antibody

eNOS - endothelial nitric oxide synthase

ESWT - Extracorporeal Shockwaves Treatment

FAOS - Foot and Ankle Outcome Scale

FFI - Foot Functional Index

NSAIDS - Non steroidal anti-inflammatory drugs

PCNA - proliferating cell nuclear antigen

SW - shock waves

VEGF - Vascular Endothelial Growth Factor

\section{Authors contributions}

FG, AB, and HM-E, ESWT assessment and manuscript drafting. CS and SM, concept and critical revision.

Acknowledgments None.

\section{Funding}

This work was supported by Elvation-Wolf by providing the shockwaves device (Piezowave). ${ }^{2}$

\section{Conflict of Interest}

None of the authors has any conflict of interest to disclose.

\section{Ethical Publication Statement}

We confirm that we have read the Journal's position on issues involved in ethical publication and affirm that this report is consistent with those guidelines.

\section{Corresponding Author}

Federico Giordani, Department of Physical and Rehabilitation Medicine, University of Padua, Padua, Italy. Phone: +390498212598

Email: federico.giordi@gmail.com

\section{E-mails of co-authors}

Andrea Bernini: andrea.bernini92@gmail.com

Hannes Müller-Ehrenberg: info@triggerpunktzentrum.de

Carla Stecco: carla.stecco@unipd.it

Stefano Masiero: stefano.masiero@unipd.it

\section{References}

1. Wearing SC, Smeathers JE, Urry SR, et al. The Pathomechanics of Plantar Fasciitis. Sport Med 2006;36:585-611.

2. Hammer DS, Adam ÆF, Kreutz ÆA. Ultrasonographic evaluation at 6-month follow-up of plantar fasciitis after extracorporeal shock wave therapy. Arch Orthop Trauma Surg 2005;125:6-9.

3. Prospective A, Trial P, Rompe JD, et al. Shock Wave Application for Chronic Plantar Fasciitis in Running Athletes. Am J Sports Med 2003;31:26875.

4. Thomson CE, Crawford F, Murray GD. The effectiveness of extra corporeal shock wave therapy for plantar heel pain : a systematic review and metaanalysis. BMC Musculoskelet Disord 2005;11:111.

5. Mitchell I, Meyer C, Krueger W. Deep fascia of the foot. Anatomical and clinical considerations. J Am Podiatr Med Assoc 1991;81(7):373-8.

6. Buchbinder R, Ptasznik R, Gordon $J$, et al. Ultrasound-Guided Extracorporeal Shock Wave Therapy for Plantar Fasciitis: a randomized controlled trial. JAMA 2002;288:1364-72.

7. Lareau CR, Sawyer GA, Wang JH, DiGiovanni CW. Plantar and Medial Heel Pain. J Am Acad Orthop Surg 2014;22:372-80.

8. Rompe JD, Hopf C, Nafe B, Bürger R. Low-energy extracorporeal shock wave therapy for painful heel: a prospective controlled single-blind study. Arch Orthop Trauma Surg 1996;115:75-9.

9. Rompe JD. Plantar Fasciopathy. Sports Med Arthrosc 2009; 17:100-4. 
10. Furey JG. Plantar fasciitis. The painful heel syndrome. J Bone Joint Surg Am 1975;57:672-3.

11. Gill. Plantar Fasciitis: Diagnosis and Conservative Management. J Am Acad Orthop Surg 1997. 2019;5:109-17.

12. Notarnicola A, Moretti B. The biological effects of extracorporeal shock wave therapy (eswt) on tendon tissue. Muscles Ligaments Tendons J 2012;2:33-7.

13. Romeo P, Lavanga V, Pagani D, Sansone V. Extracorporeal Shock Wave Therapy in Musculoskeletal Disorders: A Review. Med Princ Pract 2014;23:7-13.

14. Kearney CJ, Hsu HP, Spector M. The use of extracorporeal shock wave-stimulated periosteal cells for orthotopic bone generation. Tissue Eng Part A 2012;18:1500-8.

15. Wang C-J, Wang F-S, Yang KD, et al. Shock wave therapy induces neovascularization at the tendonbone junction. A study in rabbits. J Orthop Res 2003;21:984-9.

16. Bosch G, Mos M De, Binsbergen R, et al. The effect of focused extracorporeal shock wave therapy on collagen matrix and gene expression in normal tendons and ligaments. Equine Vet J 2009;41:33541.

17. Sukubo NG, Tibalt E, Respizzi S, et al. Effect of shock waves on macrophages: A possible role in tissue regeneration and remodeling. Int $\mathrm{J}$ Surg 2015;24:124-30.

18. Xiong Y, Wu Q, Mi B, et al. Comparison of efficacy of shock-wave therapy versus corticosteroids in plantar fasciitis: a meta-analysis of randomized controlled trials. Arch Orthop Trauma Surg 2019;139:529-36.

19. Li S, Wang K, Sun H, et al. Clinical effects of extracorporeal shock-wave therapy and ultrasoundguided local corticosteroid injections for plantar fasciitis in adults. Medicine 2018;7: 1-9.

20. Wang C, Wang F, Yang KD. Long-term Results of Extracorporeal Shockwave Treatment for Plantar Fasciitis. Am J Sports Med 2006;592-6.

21. Haake M, Böddeker IR, Decker T, et al. Side-effects of extracorporeal shock wave therapy (ESWT) in the treatment of tennis elbow. Arch Orthop Trauma Surg 2002;122(4):222-8.

22. Gerdesmeyer L1, Frey C, Vester J, et al. Radial extracorporeal shock wave therapy is safe and effective in the treatment of chronic recalcitrant plantar fasciitis: results of a confirmatory randomized placebo-controlled multicenter study. Am J Sports Med 2008;36:2100-9.

23. Purcell RL, Schroeder IG, Keeling LE, et al. Clinical Outcomes After Extracorporeal Shock Wave Therapy for Chronic Plantar Fasciitis in a Predominantly Active Duty Population. J Foot Ankle Surg 2018;57:654-7.
24. Metzner G, Dohnalek C, Aigner E. High-Energy Extracorporeal Shock-Wave Therapy (ESWT) for the Treatment of Chronic Plantar Fasciitis. Foot Ankle Int 2010;31:790-6.

25. Kudo P, Dainty K, Clarfield M, et al. Randomized, placebo-controlled, double-blind clinical trial evaluating the treatment of plantar fasciitis with an extracoporeal shockwave therapy (ESWT) device: a North American confirmatory study. J Orthop Res 2006;24:115-23.

26 Ogden JA, Alvarez R, Levitt R, et al. Shock wave therapy for chronic proximal plantar fasciitis. Clin Orthop Relat Res 2001;387:47-59.

27. Haake M, Buch M, Schoellner C, et al. Extracorporeal shock wave therapy for plantar fasciitis: randomised controlled multicentre trial. Clin Podiatr Med Surg 2003;20:1-5.

28. Speed CA, Nichols D, Wies J, et al. Extracorporeal shock wave therapy for plantar fasciitis. A double blind randomised controlled trial. J Orthop Res 2003;21:937-40.

29. Wang C-J, Chen H-S, Huang T-W. Shockwave Therapy for Patients with Plantar Fasciitis: A OneYear Follow-up Study. Foot Ankle Int 2002;23:204-7.

30. Malliaropoulos N, Crate G, Meke M, et al. Success and Recurrence Rate after Radial Extracorporeal Shock Wave Therapy for Plantar Fasciopathy: A Retrospective Study. Biomed Res Int 2016;2016: 9415827. doi: 10.1155/2016/9415827.

31. Stecco A, Stern R, Fantoni I, et al. Fascial Disorders: Implications for Treatment. Biomed Res Int 2016; 8:161-8.

32. Pascual Huerta J. The effect of the gastrocnemius on the plantar fascia. Foot Ankle Clin 2014;19:70118.

33. Shaw HM, Vázquez OT, McGonagle D, et al. Development of the human Achilles tendon enthesis organ. J Anat 2008;213:718-24.

34. Stecco C, Corradin M, Macchi V, et al. Plantar fascia anatomy and its relationship with Achilles tendon and paratenon. J Anat 2013;223:665-76.

35. Franklyn-Miller A, Falvey E, McCrory P. Fasciitis first before tendinopathy: does the anatomy hold the key? Br J Sports Med 2009;43:887-9.

36. Labovitz JM, Yu J, Kim C. The Role of Hamstring Tightness in Plantar Fasciitis. Foot Ankle Spec 2011;4:141-4.

37. Pavan PG, Pachera P, Forestiero A, Natali AN. Investigation of interaction phenomena between crural fascia and muscles by using a threedimensional numerical model. Med Biol Eng Comput 2017;55:1683-91.

38. Stecco C, Gagey O, Macchi V, et al. Tendinous muscular insertions onto the deep fascia of the upper limb. First part: anatomical study. Morphologie 2007;91:29-37. 
39. Pavan PG, Pachera P, Stecco C, Natali AN. Biomechanical behavior of human crural fascia in anterior and posterior regions of the lower limb. Med Biol Eng Comput 2015;53:951-9.

40. Renan-Ordine R, Alburquerque-SendÍn F, Rodrigues De Souza DP, et al. Effectiveness of Myofascial Trigger Point Manual Therapy Combined With a Self-Stretching Protocol for the Management of Plantar Heel Pain: A Randomized Controlled Trial. J Orthop Sport Phys Ther 2011;41:43-50.
41. Rompe JD, Furia J, Cacchio A, et al. Radial shock wave treatment alone is less ef fi cient than radial shock wave treatment combined with tissuespecific plantar fascia- stretching in patients with chronic plantar heel pain. Int J Surg 2015;24:13542.

Submission: July, 01, 2019

Revision received: August 10, 2019

Acceptance: August 10, 2019 